PROCEEDINGS OF THE

AMERICAN MATHEMATICAL SOCIETY

Volume 138, Number 7, July 2010, Pages 2489-2492

S 0002-9939(10)10328-1

Article electronically published on February 12, 2010

\title{
TANGENCIES BETWEEN HOLOMORPHIC MAPS AND HOLOMORPHIC LAMINATIONS
}

\author{
A. EREMENKO AND A. GABRIELOV \\ (Communicated by Mario Bonk)
}

\begin{abstract}
We prove that the set of leaves of a holomorphic lamination of codimension one that are tangent to a germ of a holomorphic map is discrete.
\end{abstract}

Let $F$ be a holomorphic lamination of codimension one in an open set $V$ in a complex Banach space $B$. In this paper, this means that $V=W \times \mathbf{C}$, where $W$ is a neighborhood of the origin in some Banach space, and the leaves $L_{\lambda}$ of the lamination are disjoint graphs of holomorphic functions $\mathbf{w} \mapsto f(\lambda, \mathbf{w}), W \rightarrow \mathbf{C}$. For holomorphic functions in a Banach space we refer to [5]. Here $\lambda$ is a parameter and we assume that the dependence of $f$ on $\lambda$ is continuous. A natural choice of this parameter is such that $\lambda=f(\lambda, 0)$, in which case the continuity with respect to $\lambda$ follows from the so-called $\lambda$-lemma of Mane-Sullivan-Sad and Lyubich; see, for example, 5. With this choice of the parameter, our definition of a lamination coincides with that of a holomorphic motion of $\mathbf{C}$ parametrized by $W$.

Let $\gamma: U \rightarrow V$ be a holomorphic map with $U \subset \mathbf{C}^{n}$. We say that $\gamma$ is tangent to the lamination at a point $\mathbf{z}_{0} \in U$ if the image of the derivative $\gamma^{\prime}\left(\mathbf{z}_{0}\right)$ is contained in the tangent space $T_{L}\left(\gamma\left(\mathbf{z}_{0}\right)\right)$, where $L$ is the leaf passing through $\gamma\left(\mathbf{z}_{0}\right)$. A leaf for which this holds is called a tangent leaf to $\gamma$.

Theorem. Let $K$ be a compact subset of $U$. Then the set of leaves tangent to $\gamma$ at the points of $K$ is finite.

For the case of holomorphic curves $(n=1)$ this result is contained in [1, Lemma 9.1] where it is credited to Douady. Artur Avila, in a conversation with the authors, proposed to extend this result to arbitrary holomorphic maps. According to Avila, this generalization has several applications to holomorphic dynamics [1.

Proof. We assume without loss of generality that

$$
f(0, \mathbf{w}) \equiv 0
$$

and that $L_{0}$ is tangent to $\gamma$ at $\mathbf{z}_{0}=0$.

We have to show that other tangent leaves cannot accumulate to $L_{0}$. Suppose the contrary; that is, suppose that there is a sequence $\lambda_{k} \rightarrow 0$ such that $L_{\lambda_{k}}$ are tangent to $\gamma$, and let $L_{\lambda_{k}}$ be the graphs of the functions $f_{k}(\mathbf{w})=f\left(\lambda_{k}, \mathbf{w}\right)$. We may assume that tangency points $\mathbf{z}_{k} \rightarrow 0$.

Received by the editors October 25, 2008, and, in revised form, October 28, 2009.

2010 Mathematics Subject Classification. Primary 32B99; Secondary 37F99.

The first author was supported by NSF grant DMS-0555279.

The second author was supported by NSF grant DMS-0801050.

(C)2010 American Mathematical Society Reverts to public domain 28 years from publication 
We make several preliminary reductions.

1. Let

$$
\gamma(\mathbf{z})=(\phi(\mathbf{z}), \psi(\mathbf{z})) \in W \times \mathbf{C} .
$$

Consider the new lamination in $U \times \mathbf{C}$ whose leaves are the graphs of $f^{*}(\lambda, \mathbf{z})=$ $f(\lambda, \phi(\mathbf{z}))$ and the new map $\gamma^{*}(\mathbf{z})=(\mathbf{z}, \psi(\mathbf{z}))$. Then $\gamma^{*}$ is tangent to a leaf $L^{*}$ if and only if $\gamma$ is tangent to $L$. This reduces our problem to the case where $W$ is an open set in $\mathbf{C}^{n}$ and the map $\gamma$ is a graph of a function $\psi$ of the same variable as the functions $f_{k}$. From now on we assume that $U=W$ and $\gamma(\mathbf{w})=(\mathbf{w}, \psi(\mathbf{w}))$.

2. Now we reduce the problem to the case where $\psi$ is a monomial. For this we use the desingularization theorem of Hironaka [4, 2, 3, 6.

Let $X$ be a complex analytic manifold, and let $\psi$ be an analytic function on $X$. Then there exists a complex analytic manifold $M$ and a proper surjective map $\pi: M \rightarrow X$ such that the restriction of $\pi$ onto the complement of the $\pi$-preimage of the set $\left\{\psi=0, \psi^{\prime}=0\right\}$ is injective and for each point $\mathbf{z}_{0} \in \pi^{-1}(\{\psi=0\})$ there is a local coordinate system with the origin at $\mathbf{z}_{0}$ such that $\psi \circ \pi$ is a monomial $z_{1}^{m_{1}} \ldots z_{n}^{m_{n}}$. We choose such $M$ corresponding to $X=W$.

Let $Y=W \times \mathbf{C}$, and let $S \subset Y$ be the set of points $(\mathbf{w}, t)$ with $t \neq 0$ such that the graph of $t=\psi(\mathbf{w})$ is tangent to the lamination. In our proof by contradiction, we assume that the origin belongs to the closure of $S$. Let $N=M \times \mathbf{C}$, and let $\rho: N \rightarrow Y$ be the map defined by $\rho(\mathbf{z}, t)=(\pi(\mathbf{z}), t)$. Then $\rho^{-1}(F)$ is the lamination whose leaves are the components of the $\rho$-preimages of the leaves of $F$, and the set $T=\rho^{-1}(S)$ has a limit point $\left(\mathbf{z}_{0}, 0\right)$ with $\pi\left(\mathbf{z}_{0}\right)=0$ since $\pi$ is proper. Also, the set $T$ is exactly the set of those points in $N$ where the graph $t=\psi \circ \pi(\mathbf{z})$ is tangent to the lamination $\rho^{-1}(F)$ since $\rho$ is injective in a neighborhood of each point of $T$. (Any point $(\mathbf{z}, t)$ where $\rho$ is not injective satisfies $\psi(\pi(\mathbf{z}))=0$, while at every point of $T$ we have $\psi(\pi(\mathbf{z})) \neq 0$.) This reduces our problem to the case where $\psi$ is a monomial.

3. We may assume now that $W=\{\mathbf{z}:|\mathbf{z}|<2\}$. Thus, we are in the situation

$$
\psi(z)=z_{1}^{m_{1}} \ldots z_{n}^{m_{n}},
$$

and $\left\{f_{k}\right\}$ is a family of holomorphic functions on $W$ with disjoint graphs, $f_{k}(\mathbf{z}) \neq 0$ for $\mathbf{z} \in W$, and $f_{k} \rightarrow 0$ as $k \rightarrow \infty$ uniformly on $W$. Moreover, for some sequence $\mathbf{z}_{k} \rightarrow 0$ we have

$$
\begin{gathered}
f_{k}\left(\mathbf{z}_{k}\right)=\prod_{j=1}^{n} z_{j, k}^{m_{j}}, \\
\operatorname{grad} f_{k}\left(\mathbf{z}_{k}\right)=\left(m_{1} z_{1, k}^{m_{1}-1} z_{2, k}^{m_{2}} \ldots, m_{2} z_{1}^{m_{1}} z_{2, k}^{m_{2}-1} \ldots\right),
\end{gathered}
$$

assuming zero values for the components with $m_{j}=0$. We may assume that $\left|f_{k}\right| \leq 1$ in $W$. Setting $f_{k}=\exp g_{k}$ we obtain that $\Re g_{k} \leq 0$. Now we put

$$
h_{k}(\mathbf{z})=g_{k}\left(\mathbf{z}+\mathbf{z}_{k}\right)-g_{k}\left(\mathbf{z}_{k}\right) .
$$

Then the $h_{k}$ are defined in the unit ball and satisfy

$$
\Re h_{k}(\mathbf{z}) \leq \sum_{j: m_{j}>0} m_{j} \log \left|z_{j, k}\right|^{-1}, h_{k}(0)=0 .
$$


From this we conclude that

$$
\left|\frac{\partial h_{k}}{\partial z_{j}}(0)\right| \leq 2 \sum_{j: m_{j}>0} m_{j} \log \left|z_{j, k}\right|^{-1} .
$$

This follows from the following lemma.

Lemma. Let $h$ be an analytic function in the unit disc, $h(0)=0$, and $\Re h \leq A$, where $A>0$. Then $\left|h^{\prime}(0)\right| \leq 2 A$.

Proof. The function

$$
\phi(z)=\frac{z}{2 A z-z}
$$

maps the half-plane $\{\Re z<A\}$ onto the unit disc, $\phi(0)=0$, and $\phi^{\prime}(0)=1 /(2 A)$. Now the statement follows from the Schwarz lemma applied to $\phi \circ h$ and the chain rule:

$$
\left.\left|(\phi \circ h)^{\prime}(0)\right|=\left|\phi^{\prime}(0) h^{\prime}(0)\right|=\mid h^{\prime}(0) / 2 A\right) \mid \leq 1 .
$$

On the other hand, (11) and (2) imply that, for $m_{j}>0$,

$$
\left|\frac{\partial h_{k}}{\partial z_{j}}(0)\right|=\frac{m_{j}}{\left|z_{j, k}\right|} \text {. }
$$

Assume without loss of generality that $m_{1}>0$ and

$$
\left|z_{1, k}\right|=\min _{j: m_{j}>0}\left|z_{j, k}\right| \text {. }
$$

Then the right-hand side of (3) is at most

$$
\text { const } \log \left|z_{1, k}\right|^{-1}
$$

while the right-hand side of (4) for $j=1$ is

$$
\frac{m_{1}}{\left|z_{1, k}\right|} \text {. }
$$

As $\left|z_{1, k}\right| \rightarrow 0$, we obtain a contradiction which proves our theorem.

\section{ACKNOWLEDGMENT}

We thank the referee for useful comments.

\section{REFERENCES}

[1] A. Avila, M. Lyubich and W. de Melo, Regular or stochastic dynamics in real analytic families of unimodal maps, Invent. Math. 154 (2003), no. 3, 451-550. MR2018784 (2006i:37083)

[2] E. Bierstone and P.D. Milman, Canonical desingularization in characteristic zero by blowing up the maximum strata of a local invariant, Invent. Math. 128 (1997), 207-302. MR 1440306 (98e:14010)

[3] S. Encinas and O. Villamayor, A new proof of desingularization over fields of characteristic zero, Revista Matematica Iberoamericana 19 (2003), 339-353. MR.2023188 (2004m:14017)

[4] H. Hironaka, Resolution of singularities of an algebraic variety over a field of characteristic zero. I, Ann. of Math. (2) 79 (1964), 109-203; II, ibid. (2) 79 (1964), 205-326. MR0199184 (33:7333) 
[5] J. Hubbard, Teichmüller theory and applications to geometry, topology, and dynamics, Vol. 1, Matrix Editions, Ithaca, NY, 2006. MR2245223 (2008k:30055)

[6] J. Wlodarczyk, Simple Hironaka resolution in characteristic zero, J. Amer. Math. Soc. 18 (4) (2005), 779-822. MR2163383 (2006f:14014)

Department of Mathematics, Purdue University, West Lafayette, Indiana 47907

E-mail address: eremenko@math.purdue.edu

Department of Mathematics, Purdue University, West Lafayette, Indiana 47907

E-mail address: agabriel@math.purdue.edu 